\title{
Formulae and Growth Rates of High-Dimensional Polycubes
}

\author{
Ronnie Barequet* Gill Barequet ${ }^{\dagger} \quad$ Günter Rote R $^{\ddagger}$
}

\begin{abstract}
A $d$-dimensional polycube is a facet-connected set of cubes in $d$ dimensions. Fixed polycubes are considered distinct if they differ in their shape or orientation. A proper $d$-dimensional polycube spans all the $d$ dimensions, that is, the convex hull of the centers of its cubes is $d$-dimensional. In this paper we prove rigorously some (previously conjectured) closed formulae for fixed (proper and improper) polycubes, and show that the growth-rate limit of the number of polycubes in $d$ dimensions is $2 e d-o(d)$. We conjecture that it is asymptotically equal to $(2 d-3) e+O(1 / d)$.
\end{abstract}

Keywords: Polyominoes, lattice animals.

\section{Introduction}

A $d$-dimensional polycube of size $n$ is a connected set of $n$ cubical cells on the lattice $\mathbb{Z}^{d}$, where connectivity is through $(d-1)$-faces. Two fixed polycubes are considered equivalent if one can be transformed into the other by a translation. (In contrast, two free polycubes are considered equivalent if one can be transformed into the other by a translation, flip, and/or rotation.) A fixed polycube is called improper in $d$ dimensions if the centers of all its cubes lie in a common hyperplane of dimension strictly less than $d$.

In the literature of statistical physics, fixed polycubes are usually referred to as stronglyembedded lattice site animals. To understand this term, consider the dual graph of the hypercubic lattice. The dual entities of a cell and an adjacency relation of two cells are a vertex and an edge (bond) connecting two cells, respectively. An "animal" is a connected subgraph (cluster) of vertices. A weakly-embedded animal may contain nearest-neighbor

*Dept. of Mathematics and Dept. of Computer Science, Tel Aviv University, Tel Aviv 69978, Israel. E-mail: ronnieba@post.tau.ac.il

${ }^{\dagger}$ Dept. of Computer Science, The Technion-Israel Institute of Technology, Haifa 32000, Israel. E-mail: barequet@cs.technion.ac.il

${ }_{\ddagger}^{\ddagger}$ Institut für Informatik, Freie Universität Berlin, Takustraße 9, D-14195 Berlin, Germany. E-mail: rote@inf.fu-berlin.de 
lattice vertices but not the bond that connects between them. The size of a site (resp., bond) animal is the number of vertices (edges) it contains. A tree animal is a subgraph that does not contain any cycles.

Counting polyominoes (2-dimensional polycubes) is a long-standing problem in discrete geometry, originating in statistical physics [BH57] and having many applications, e.g., in cryptography [SE05]. The sequence $A(n)$, which lists the number of fixed polyominoes, is currently known up to $n=56$ [Je03]. Enumerating polycubes in higher dimensions is an even more elusive problem. Lunnon [Lu72] manually counted 3-dimensional polycubes (up to size 6) by considering symmetry groups. In a subsequent work, Lunnon [Lu75] computed the number of small-sized polycubes in up to 6 dimensions. Aleksandrowicz and Barequet [AB06, AB08] extended polycube counting by efficiently generalizing Redelmeier's polyomino-counting algorithm [Re81] to higher dimensions.

There is an extensive statistical-physics literature which provides enumeration data of polycubes. Gaunt, Sykes, and Ruskin [GSR76] provided the numbers of 3- to 7-dimensional polycubes up to size 13,11, 10, 9, and 9, respectively (with a slight error in the count of 3-dimensional polycubes of size 13). The counts of 3-dimensional polycubes of up to size 17 can be derived from data obtained by Martin [Ma90] and published by Madras et al. [MSW+90]. Gaunt and Peard [GP00, p. 7520] claimed to have (and to present in a further publication) the counts of 3 -dimensional polycubes of up to size 19, but we were unable to locate these numbers. Gaunt [Ga80] provided the counts of polycubes of size up to 9 in 8 and 9 dimensions.

The growth-rate limit of polyominoes in the plane has also attracted much attention in the literature. Klarner [Kl67] showed that the limit (also called Klarner's constant) $\lambda_{2}=\lim _{n \rightarrow \infty} \sqrt[n]{A(n)}$ exists, although its exact value is to date unknown. The convergence of $A(n+1) / A(n)$ to $\lambda_{2}$, as $n$ tends to infinity, was proved only 32 years later by Madras [Ma99]. The best-known lower [BMRR06] and upper [KR73] bounds on $\lambda_{2}$ are 3.9801 and 4.6496, respectively. It is generally assumed [GSR76, Ga80, GPSW94, Je03], as a conclusion from numerical methods applied to the known values of $A(n)$, that $\lambda_{2} \approx 4.06$. In $d>2$ dimensions, the growth-rate limit $\lambda_{d}$ of the number of polycubes is also guaranteed to exist [Ma99].

In the statistical-physics literature there are various models of animals (site or bond, weakly- or strongly embedded, general or trees, etc.), and the asymptotic growth rate of animals is called the "reduced limiting free energy." When referring to the strongly-embedded site animals model, this quantity is usually denoted by the symbol $\Lambda_{s}$ (or $\Lambda_{s}^{(d)}$ to indicate the dimension). There are many works (e.g., [GSR76, GPSW94, PG95, GP00]) on representations of $\ln \Lambda_{s}$ (our $\ln \lambda_{d}$ ), as well as the respective growth constants of other models of animals, as a power series in $1 / d$, so-called the $1 / d$-expansion of the free energy of animals. As described in more detail in Section 5, the most accurate estimate, that we are aware of, is (see $[\mathrm{GP} 00]$ )

$$
\ln \lambda_{d}=\ln \sigma+1-\frac{2}{\sigma}-\frac{79}{24 \sigma^{2}}-\frac{317}{24 \sigma^{3}}-\frac{18321}{320 \sigma^{4}}-\frac{123307}{240 \sigma^{5}}+O\left(\frac{1}{\sigma^{6}}\right),
$$

where $\sigma=2 d-1$ is the coordination number of the lattice. Such expansions are unrigorous 
since they depend on previously-unproven formulae, e.g., the formula that we prove in Theorem 6 (see Section 3). It is commonly said that these expansions seem to be "asymptotic but not convergent." In the last section of this paper we show how this unrigorous expansion supports our conjecture that $\lambda_{d} \sim(2 d-3) e$ as $d$ tends to infinity.

In this paper, we follow Lunnon's notation [Lu75], denoting by $\operatorname{DX}(n, d)$ the number of proper fixed polycubes of size $n$ in $d$ dimensions, and by $\mathrm{CX}(n, d)$ the number of all (proper and improper) such polycubes. In the mathematical literature on counting high-dimensional polycubes, the value of $d$ is normally fixed and the functions $\operatorname{CX}(n, d)$ and $\operatorname{DX}(n, d)$ are investigated as functions of $n$ only. As mentioned above, the function $\mathrm{CX}(n, 2)$ is usually denoted in the literature as $A(n)$. Similarly, when the dimension $d$ is fixed, the function $\mathrm{CX}(n, d)$ is denoted in $[\mathrm{AB} 06, \mathrm{AB} 08]$ as $A_{d}(n)$. In Section 4 we fix the value of $n$ and investigate $\mathrm{DX}(n, d)$ and $\mathrm{CX}(n, d)$ as functions of $d$ only. For clarity of notation, we denote these functions as $\mathrm{CX}_{n}(d)$ and $\operatorname{DX}_{n}(d)$, respectively. In the statistical-physics literature, these functions have several notations, e.g., $D_{n}(d)$ [GSR76, pp. 1900-1901] (the parameter $q$ in the formula should be substituted by 1 to count polycubes), $Z_{n}(z=0, k)$ [PG95, App. 1] as a function of $d$ (the parameter $z$ should be substituted by 0 to count site animals, and ' $k$ ' is an attribute that means the strongly-embedded animals model), $Z_{n}(y=1, z=0 ; c, k)$ [GP00, App. A] as a function of $d(z=0$ as above, and $y$ should be substituted by 1 to count all animals and not only trees, while $c$ and $k$ refer to the model), etc.

The paper is organized as follows. In Section 2 we review Lunnon's formula that relates the total number of polycubes to the number of proper polycubes. In Section 3 we develop a few diagonal formulae for the number of proper polycubes, in which the difference between the size and the dimension is at most 3. (Surprisingly, these formulae bear great resemblance to Chebyshev polynomials.) In Section 4 we show that when the size of the polycubes is fixed, the number of polycubes is a ("row") polynomial in the dimension; we compute the first ten row polynomials. Based on the diagonal formulae, we also compute formulae for the first three sequences of coefficients of these polynomials. In Section 5 we show, based on the row polynomials and the coefficient sequences, that the limit growth rate of the number of polycubes, as $d$ tends to infinity, is roughly $(2 d-3) e$. We end in Section 6 with some concluding remarks.

\section{Lunnon's Formula}

Lunnon [Lu75, p. 366] observed the following relation between $\operatorname{CX}(n, d)$ and $\operatorname{DX}(n, d)$ :

$$
\mathrm{CX}(n, d)=\sum_{i=0}^{d}\left(\begin{array}{l}
d \\
i
\end{array}\right) \operatorname{DX}(n, i) .
$$

This is easily justified by summing up all proper polycubes in all dimensions. In $0 \leq$ $i \leq d$ dimensions, each of the $\operatorname{DX}(n, i)$ proper $i$-dimensional polycubes contributes $\left(\begin{array}{l}d \\ i\end{array}\right) \bar{d}$ dimensional polycubes by enumerating the spanned dimensions. In case $n \leq d$, all the respective terms of $i>n-1$ are zero since $\mathrm{DX}(n, i)=0$ (see Section 3). Note that for 


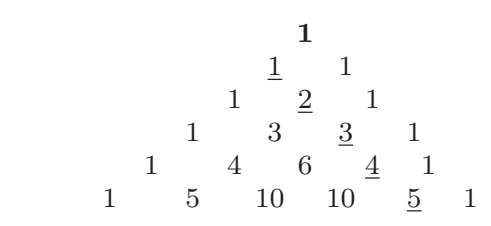

(a) $n=2$

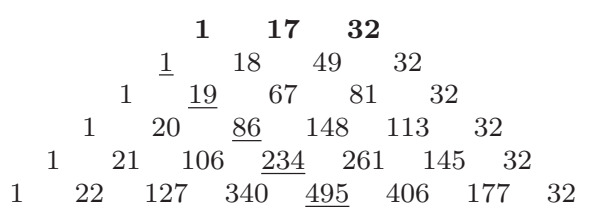

(c) $n=4$

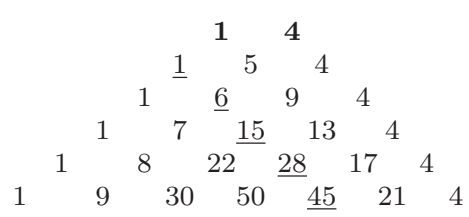

(b) $n=3$

Figure 1: Lunnon's formula in a Pascal trapezoid

consistency, $\mathrm{DX}(1,0)=1$ and $\mathrm{DX}(n, 0)=0$ for $n \geq 2$ (only the polycube of size 1 is said to be proper in zero dimensions); thus, we also have $\mathrm{CX}(1,0)=1$ and $\mathrm{CX}(n, 0)=0$ for $n \geq 2$. To summarize, we can rewrite Lunnon's formula as

$$
\mathrm{CX}(n, d)=\sum_{i=0}^{\min (n-1, d)}\left(\begin{array}{l}
d \\
i
\end{array}\right) \operatorname{DX}(n, i) .
$$

One can visualize Lunnon's formula in a Pascal triangle-like construction. Figure 1 shows this trapezoidal diagram for a few small values of $n$. For a fixed value of $n$, the first line of the trapezoid contains the values of $\operatorname{DX}_{n}(d)$ for $1 \leq d \leq n-1$ (seen in boldface). The values of $\mathrm{CX}_{n}(d)$ (underlined in the figure) are revealed in the $n$th left-to-right diagonal of the trapezoid.

Lunnon's formula has been widely used in the statistical-physics literature on lattice animals, and is called a "partition formula," where the partition is usually according to a few more parameters (attributes of the polycubes). The earliest references for this, that we are aware to, are from the 1960s.

\section{Diagonals, or: Minimal Proper Polycubes}

Obviously, we have $\operatorname{DX}(n, d)=0$ for $n<d+1$. This is because the center of a single cube occupies zero dimensions, and each additional cube increases the dimensionality of the connectivity graph of the centers of the cubes of the polycube by either 0 or 1 . That is, polycubes of size $n$ cannot be proper in more than $n-1$ dimensions. Equivalently, proper $d$-dimensional polycubes must consist of at least $d+1$ cubes. Lunnon [Lu75] computed $\operatorname{DX}(n, n-1)$ up to $n=7$. However, as already noted elsewhere (e.g., in [GSR76, AB06, AB08]), he specified an incorrect value for $\operatorname{DX}(7,6)$. Gaunt [Ga80] provided values of $\operatorname{DX}(n, n-1)$ up to $n=9$. The number of these minimal proper polycubes is given by the following theorem.

Theorem 1. $\operatorname{DX}(n, n-1)=2^{n-1} n^{n-3}$. 
This formula is usually justified by the fact that these minimal proper polycubes are in one-to-one correspondence with Cayley trees. Many works mention it either implicitly of explicitly; see, e.g., [GSR76, Eq. (2.3)] and [PG95, Eq. (2.9)]. For completeness we provide here a full proof of it. It is more convenient to use trees that carry labels on the edges rather than the vertices.

Lemma 2. The number of directed trees with $n$ vertices and $n-1$ distinct edge labels $1, \ldots, n-1$ is $2^{n-1} n^{n-3}$, for $n \geq 2$.

In our context, "directed" means that each edge has an arbitrary orientation, in addition to its label. Thus, the orientations simply contribute the factor $2^{n-1}$.

Proof. The number of undirected trees with vertex labels $0,1, \ldots, n-1$ (Cayley trees) is $n^{n-2}$, and with directed edges we get $2^{n-1} n^{n-2}$. In such a tree, we declare vertex 0 to be the root and "shift" each remaining vertex label to the incident edge towards the root. In this way, we get $2^{n-1} n^{n-2}$ edge-labeled directed trees as in the lemma, with an additional marked "root" vertex 0 . Since there are $n$ distinct choices for this root vertex, we have to divide by $n$, to get $2^{n-1} n^{n-3}$ trees without a vertex marked.

Proof of Theorem 1. We show that the edge-labeled directed trees on $n$ vertices of Lemma 2 are in one-to-one correspondence with the proper $(n-1)$-dimensional polycubes of size $n$.

Starting with such a polycube $P$, we can take the adjacency graph $G$ of cubes in $P$. We label each edge by the dimension in which the two cubes are adjacent (i.e., the unique coordinate in which the two cubes differ by \pm 1 ), and we orient the edge from the smaller coordinate to the larger coordinate. By construction, the graph $G$ is connected.

We claim that it is a tree. To see this, consider a spanning tree $T$ of $G$. Its $n-1$ edges are labeled by $1, \ldots, n-1$, and each label occurs at least once. Otherwise, if some label $k$ were missing, all cubes would have the same $k$ th coordinate, and hence the polycube $P$ would not span all $n-1$ dimensions. It follows that each label $1, \ldots, n-1$ occurs exactly once. From this we can infer that two cubes $u$ and $v$ that are not adjacent in $T$ are also not adjacent in $G$, and hence $G=T$ : the difference in coordinates between $u$ and $v$ can be calculated by following the labels on the path between $u$ and $v$, and if this path contains $l \geq 2$ edges, the coordinates differ in $l$ positions.

We have established that each polycube produces an edge-labeled directed tree $T$. Conversely, if we are given such a tree $T$, we can reverse the process and build the corresponding polycube $P$ as follows: we start from any node as a singleton cube, and search $T$ (say, using depth-first search). We attach a new lattice cube for each new vertex in the proper dimension and the direction (positive or negative) as implied by the label and orientation of the edge. This results in a valid polycube $P$ with $n$ cubes. It is connected and proper in $n-1$ dimensions, by construction. To see that no two cubes in $P$ "overlap," we can use the argument above which showed that no two cubes (non-adjacent in $T$ ) can be adjacent. Much less can two such cubes coincide. 
Corollary 3. The limit growth rate of the number of minimal proper polycubes (divided by the size of the polycubes) is $2 e$.

Proof.

$$
\frac{\mathrm{DX}(n+1, n) / \mathrm{DX}(n, n-1)}{n+1}=\frac{2^{n}(n+1)^{n-2}}{2^{n-1} n^{n-3}(n+1)}=2\left(\frac{n+1}{n}\right)^{n-3} \underset{n \rightarrow \infty}{\longrightarrow} 2 e .
$$

Formulae for $\mathrm{DX}(n, n-2)$ and $\mathrm{DX}(n, n-3)$ can be extrapolated from the known values of DX. In statistical physics, they are widely assumed to be true, but have never been proven rigorously. We provide here a full proof of the formula for the second diagonal. First, we prove a lemma and corollary which generalize Lemma 2.

Lemma 4. The number of ordered sequences of $k \geq 1$ rooted trees with a total of $n-k$ edges and distinct edge labels $1, \ldots, n-k$ is $n^{n-k-1} k$.

By "rooted" we mean that each tree in the forest has a distinguished marked vertex.

Proof. Recall (see, e.g., [Mo70]) that the number of labeled trees with $n$ vertices of given degrees $d_{1}, \ldots, d_{n}$ is $\left(\begin{array}{c}n-2 \\ d_{1}-1, \ldots, d_{n}-1\end{array}\right)$.

In our case, we look at vertex-labeled trees with $n+1$ nodes, in which an auxiliary "root" node (labeled $n+1$ ) has degree $k$. Fixing $d_{n+1}=k$ and summing over all possible $d_{1}, d_{2}, \ldots, d_{n}$, using the above formula, yields the number of such trees. We are interested in the number $X$ of trees in which the $k$ neighbors of vertex $n+1$ have labels $n, n-1, n-2, \ldots, n-k+1$. Since all $\left(\begin{array}{l}n \\ k\end{array}\right)$ subsets of labels are equally likely to occur as labels of the root neighbors, we divide by the binomial coefficient $\left(\begin{array}{l}n \\ k\end{array}\right)$ :

$$
\begin{aligned}
X=\frac{\sum_{\substack{d_{1}, \ldots, d_{n} \\
d_{1}+\cdots+d_{n}=2 n-k}}\left(\begin{array}{c}
n-1 \\
d_{1}-1, \ldots, d_{n}-1, k-1
\end{array}\right)}{\left(\begin{array}{l}
n \\
k
\end{array}\right)} & =\frac{\left(\begin{array}{l}
n-1 \\
k-1
\end{array}\right)}{\left(\begin{array}{l}
n \\
k
\end{array}\right)} \cdot \sum_{\substack{d_{1}, \ldots, d_{n} \\
d_{1}+\cdots+d_{n}=2 n-k}}\left(\begin{array}{c}
n-k \\
d_{1}-1, \ldots, d_{n}-1
\end{array}\right) \\
& =\frac{k}{n} \cdot \sum_{\substack{e_{1}, \ldots, e_{n} \\
e_{1}+\cdots+e_{n}=n-k}}\left(\begin{array}{c}
n-k \\
e_{1}, \ldots, e_{n}
\end{array}\right) \\
& =\frac{k}{n} \cdot n^{n-k}=n^{n-k-1} k .
\end{aligned}
$$

The resulting trees are in one-to-one correspondence with the trees of the lemma, discarding vertex $n+1$, using vertices $n, n-1, n-2, \ldots, n-k+1$ as the ordered sequence of roots, and shifting vertex labels to the incident edges as in the proof of Lemma 2.

Corollary 5. The number of ordered sequences of $k \geq 1$ directed rooted trees with a total of $n-k$ edges and distinct edge labels $1, \ldots, n-k$ is $2^{n-k} n^{n-k-1} k$.

Theorem 6. DX $(n, n-2)=2^{n-3} n^{n-5}(n-2)\left(2 n^{2}-6 n+9\right)$. 
Proof. Every proper polycube of size $n$ in $n-2$ dimensions has one or more spanning tree. (Multiplicity is caused by possible cycles.) As in the proof of Theorem 1, a spanning tree is encoded as a directed edge-labeled tree on $n$ nodes with labels from the set $\{1, \ldots, n-2\}$, where each label (dimension) appears at least once. Hence, there is precisely one label $i$ that appears twice, while all other labels appear once. To avoid confusion, we use two distinct labels $i$ and $i^{\prime}$ for the two edges in direction $i$. Thus, we get spanning trees with $n-1$ distinct edge labels $1, \ldots, n-2$ and $i^{\prime}$, for some $i \in\{1, \ldots, n-2\}$. We have $n-2$ choices for the duplicated label $i$, and, thus, we get

$$
(n-2) 2^{n-1} n^{n-3}
$$

trees. Every spanning tree of a polycube is represented twice since exchanging labels $i$ and $i^{\prime}$ leads to the same tree. Moreover, in contrast to the case of Theorem 1, this count suffers from two types of errors:

(I) Some polycubes have more than one spanning tree.

(II) Some trees encode invalid (self-overlapping) polycubes.

Let us correct these counting errors. First, consider errors of type I. Polycubes with multiple trees must contain a path of the form $\bullet \frac{i}{-} \bullet \frac{j}{\bullet} \stackrel{i^{\prime}}{\bullet}$ in order to close a cycle. The four vertices marked with ' $\bullet$ ' form a quadrilateral $Q$ lying in dimensions $i$ and $j$. Fix a pair $\{i, j\}$ with $i \neq j$. This can be done in $\left(\begin{array}{c}n-2 \\ 2\end{array}\right)$ ways. Removing the four edges of $Q$ breaks the polycube into a sequence of four directed rooted spanning trees with a total of $n-4$ edges carrying distinct labels from the set $\{1, \ldots, n-2\} \backslash\{i, j\}$. According to Corollary 5, the number of such sequences is $2^{n-2} n^{n-5}$.

This is the number of polycubes that contain the square block $Q$ : one can simply attach the four tree roots to the four vertices of $Q$, taking the latter in some fixed order. On the other hand, let us check how often we have counted spanning trees that correspond to this polycube in the expression (2). The quadrilateral $Q$ has two edges in both directions $i$ and $j$. There are two choices of omitting one $j$-edge from $Q$, and two ways of distributing the labels $i$ and $i^{\prime}$ over the two $i$-edges, amounting to four possibilities. In addition, there are two choices of omitting one $i$-edge from $Q$, and two ways of distributing the labels $j$ and $j^{\prime}$ over the two $j$-edges. Thus, each polycube is represented eight times but should be counted twice as mentioned above. To recap, in order to correct errors of type I we have to subtract

$$
\left(\begin{array}{c}
n-2 \\
2
\end{array}\right) \times 6 \times 2^{n-2} n^{n-5}=3(n-2)(n-3) 2^{n-2} n^{n-5} .
$$

Second, consider errors of type II. Trees that lead to self-overlapping polycubes must contain a path of either the form $\bullet \stackrel{i}{\longleftarrow} \bullet \stackrel{i^{\prime}}{\longrightarrow} \bullet$ or the form $\bullet \stackrel{i}{\longrightarrow} \bullet \stackrel{i^{\prime}}{\longleftarrow} \bullet$. This gives us two choices. Fix $i$ (in one of $n-2$ choices). Again, according to Corollary 5 , the number of sequences of three directed rooted spanning trees with $n-3$ edges carrying distinct labels from the set $\{1, \ldots, n-2\} \backslash\{i\}$ is $3 \cdot 2^{n-3} n^{n-4}$. Attaching these trees to the three nodes in 
the above patterns creates all trees with self-overlap. To recap, in order to correct errors of type II we have to subtract

$$
2(n-2) \times 3 \cdot 2^{n-3} n^{n-4}=3(n-2) 2^{n-2} n^{n-4} .
$$

Now we can complete the computation of the correct number of polycubes under consideration:

$$
\begin{aligned}
\frac{(n-2) 2^{n-1} n^{n-3}-3(n-2)(n-3) 2^{n-2} n^{n-5}-3(n-2)}{2} 2^{n-2} n^{n-4} & \\
2 & =2^{n-3} n^{n-5}(n-2)\left(2 n^{2}-6 n+9\right),
\end{aligned}
$$

where the division by 2 is due to the double-counting mentioned in the beginning.

The above approach can be extended to proper cubes in $n-3$ dimensions, leading to the formula for the third diagonal:

$$
\operatorname{DX}(n, n-3)=2^{n-6} n^{n-7}(n-3)\left(12 n^{5}-104 n^{4}+360 n^{3}-679 n^{2}+1122 n-1560\right) / 3 .
$$

The proof is similar in nature to that of Theorem 6 but it consists of a more systematic and much more involved case analysis, and will be given in a future work. This formula predicted that $\mathrm{DX}(10,7)=17,239,040,000$. It took $91 \mathrm{CPU}$ days for the parallel version of the polycube-counting program [AB08] to confirm this value. To the best of our knowledge, this is the first verification of this number by direct enumeration of polycubes.

Conjectured formulae for the diagonal formulae $\mathrm{DX}(n, n-i)$ up to $i=6$ appear in [PG95]. There, Equation (2.15) [ibid., p. 6113] specifies $f_{i, k}(n)=2^{n-2 i+1} n^{n-2 i-1} g_{i, k}(n)$, where $k$ is the number of contacts, and the polynomials $g_{i, k}(n)$ are listed in App. 2 [ibid., p. 6120]: Since in strongly-embedded polycubes $k=0$, we have $\operatorname{DX}(n, n-i)=g_{i, 0}(n)$. The same polynomials are further parameterized by $c$, the cyclomatic index, in [GP00]. Similarly, Equation (3.4) [ibid., p. 7520] specifies $f_{i, c, k}(n)=2^{n-2 i+1} n^{n-2 i-1} g_{i, c, k}(n)$, where the polynomials $g_{i, c, k}(n)$ are listed in App. B [ibid., p. 7533]: one should ignore the polynomials with $k>0$, and have $\operatorname{DX}(n, n-i)=\sum_{c} g_{i, c, 0}(n)$.

Corollary 7. The limit growth rate of the number of polycubes along the second and third diagonals (divided by the size of the polycubes) is $2 e$.

Proof.

$$
\begin{aligned}
\frac{\mathrm{DX}(n+2, n)}{\operatorname{DX}(n+1, n-1)} /(n+2) & =\frac{2^{n-1}}{2^{n-2}} \cdot \frac{(n+2)^{n-3}}{(n+1)^{n-4}} \cdot \frac{n}{n-1} \cdot \frac{2(n+2)^{2}-6(n+2)+9}{2(n+1)^{2}-6(n+1)+9} \cdot \frac{1}{n+2} \\
& =2 \cdot\left(\frac{n+2}{n+1}\right)^{n-4} \cdot \frac{n}{n-1} \cdot \frac{2(n+2)^{2}-6(n+2)+9}{2(n+1)^{2}-6(n+1)+9} \\
& \underset{n \rightarrow \infty}{\longrightarrow} 2 \cdot e \cdot 1 \cdot 1=2 e .
\end{aligned}
$$

Similarly, it is easily verified that

$$
\frac{\mathrm{DX}(n+3, n) / \mathrm{DX}(n+2, n-1)}{n+3} \underset{n \rightarrow \infty}{\longrightarrow} 2 e .
$$


The conjectured formulae for $\operatorname{DX}(n, n-i)(4 \leq i \leq 6)$ follow the same pattern. We conjecture that the limit growth rate of the number of polycubes along any diagonal (divided by the size of the polycubes) is $2 e$; that is, $(\mathrm{DX}(n+i, n) / \mathrm{DX}(n+i-1, n-1)) /(n+i) \underset{n \rightarrow \infty}{\longrightarrow} 2 e$ for all $i>0$. In fact, this is a simple consequence of the conjectured formula $\operatorname{DX}(n, n-i)=$ $2^{n-2 i+1} n^{n-2 i-1} P_{i}(n)$, where $P_{i}(n)$ is a polynomial in $n$. The conjectured diagonal formulae imply that the degree of $P_{i}(n)$ is $3 i-4$, but this has not been proven either.

\section{Rows, or: Polycubes of Fixed Size}

In this section we show that for any fixed value of $n$, the function $\operatorname{CX}_{n}(d)$ is a polynomial in $d$. Gaunt, Sykes, and Ruskin [GSR76, §2] listed these polynomials up to $n=7$ in a Lunnon-like form: one should substitute $q:=1$ in the polynomials $D_{n}(d)$ to obtain $\mathrm{CX}_{n}(d)$. Similarly, Peard and Gaunt [PG95, App. 1] listed implicitly these polynomials up to $n=8:{ }^{1}$ one should substitute $z:=1$ in the polynomial $Z_{n}(z ; k)$ ( $k$ ' means the site model, and $z=1$ stands for strongly-embedded animals) to obtain $\mathrm{CX}_{n}(d)$. In a similar manner, one can deduce these polynomials up to $n=13$ from [GP00, App. A]: one should substitute $y:=1$ and $z:=1$ in the polynomial $Z_{n}(y, z ; c, k)$ ('c' and ' $k$ ' indicate the model, $y=1$ for counting sites, and $z=1$ for strongly-embedded animals) to obtain $\mathrm{CX}_{n}(d)$. All these implicit formulae are based on other conjectured formulae, but they agree with all known counts of polycubes. We provide below the explicit polynomials for $n \leq 10$ (which we know for sure), and also compute the formulae for the first three sequences of the leading coefficients of these polynomials. The latter sequences will prove to be beneficial in the next section.

Trivially, $\mathrm{CX}_{1}(d)=1$. It is also obvious that $\mathrm{CX}_{2}(d)=d$ : a domino can be aligned with any of the $d$ dimensions. It is also rather simple to count $d$-dimensional trominoes. A tromino can be either a "stick" or "L-shaped." There are $d$ sticks, while the number of L-shaped trominoes is $4\left(\begin{array}{c}d \\ 2\end{array}\right)$ (there are four orientations of the L-shape in each pair of spanned dimensions). In total, there are $\mathrm{CX}_{3}(d)=d+4\left(\begin{array}{l}d \\ 2\end{array}\right)=2 d^{2}-d$ trominoes. Note that $\mathrm{CX}_{3}(d)$ is also the dth hexagonal number (sequence A000384 in [Sl]).

In general, we have:

Theorem 8. $\mathrm{CX}_{n}(d)$ is a polynomial in $d$ of degree $n-1$.

Proof. The claim follows from (1). The quantity $\mathrm{CX}(n, d)$ is the sum of $\min (n-1, d)$ nonzero terms. For a fixed value of $n$, all these terms are polynomials in $d$, of which the one of the highest degree is $\operatorname{DX}(n, n-1)\left(\begin{array}{c}d \\ n-1\end{array}\right)$. The respective degree of $d$ is, thus, $n-1$.

Note that:

- For $n \geq 2, d=0$ is a root of $\operatorname{CX}_{n}(d)$ (i.e., $\left.\operatorname{CX}_{n}(0)=0\right)$. This follows from the fact that $\mathrm{CX}(n, 0)=0$ for $n \geq 2$.

\footnotetext{
${ }^{1}$ The coefficient of the last term in the formula for $n=8$ contains a typo: it should be 4,194,304 rather than 4,194,309.
} 


\begin{tabular}{|c|l|}
\hline Function & Polynomial \\
\hline $\mathrm{CX}_{1}(d)$ & 1 \\
$\mathrm{CX}_{2}(d)$ & $d$ \\
$\mathrm{CX}_{3}(d)$ & $2 d^{2}-d$ \\
$\mathrm{CX}_{4}(d)$ & $\frac{16}{3} d^{3}-\frac{15}{2} d^{2}+\frac{19}{6} d$ \\
$\mathrm{CX}_{5}(d)$ & $\frac{50}{3} d^{4}-42 d^{3}+\frac{239}{6} d^{2}-\frac{27}{2} d$ \\
$\mathrm{CX}_{6}(d)$ & $\frac{288}{5} d^{5}-216 d^{4}+\frac{986}{3} d^{3}-231 d^{2}+\frac{926}{15} d$ \\
$\mathrm{CX}_{7}(d)$ & $\frac{9604}{45} d^{6}-1078 d^{5}+\frac{20651}{9} d^{4}-\frac{14927}{6} d^{3}+\frac{120107}{90} d^{2}-\frac{827}{3} d$ \\
$\mathrm{CX}_{8}(d)$ & $\frac{262144}{315} d^{7}-\frac{26624}{5} d^{6}+\frac{132320}{9} d^{5}-\frac{65491}{3} d^{4}+\frac{1615991}{90} d^{3}-\frac{113788}{15} d^{2}+\frac{52589}{42} d$ \\
$\mathrm{CX}_{9}(d)$ & $\frac{118098}{35} d^{8}-26244 d^{7}+\frac{447903}{5} d^{6}-\frac{511082}{3} d^{5}+\frac{23014949}{120} d^{4}-\frac{1522261}{12} d^{3}$ \\
& $\quad+\frac{38839021}{840} d^{2}-\frac{30089}{4} d$ \\
$\mathrm{CX}_{10}(d)$ & $\frac{8000000}{567} d^{9}-\frac{2720000}{21} d^{8}+\frac{14272000}{27} d^{7}-\frac{11092360}{9} d^{6}+\frac{239850598}{135} d^{5}-\frac{14606026}{9} d^{4}$ \\
& $\quad+\frac{1067389643}{1134} d^{3}-\frac{42595493}{126} d^{2}+\frac{2804704}{45} d$ \\
\hline
\end{tabular}

Table 1: Explicit representations of $\mathrm{CX}_{n}(d)=a_{n, 0} d^{n-1}+a_{n, 1} d^{n-2}+\cdots+a_{n, n-2} d$

- For $n \geq 1$, the sum of coefficients of $\mathrm{CX}_{n}(d)$ is 1 (i.e., $\mathrm{CX}_{n}(1)=1$ ). Obviously, there is only one 1-dimensional polycube of any size $n$.

For a fixed value of $n$, the polynomial $\mathrm{CX}_{n}(d)$ can be computed in two ways:

1. If all values of $\mathrm{DX}(n, d)$ are known for $1 \leq d \leq n-1$ (i.e., all the nonzero values of $\mathrm{DX}(n, d))$, one can manipulate Equation (1) and obtain a representation of $\mathrm{CX}_{n}(d)$ as a polynomial in $d$; or

2. Since $\mathrm{CX}_{n}(d)$ is a polynomial in $d$ of degree $n-1$, it can be deduced from the first (or any) $n$ known values of $\operatorname{CX}(n, d)$ (including $\operatorname{CX}(n, 0)=0$ and $\operatorname{CX}(n, 1)=1$ ).

Table 1 shows explicit representations of the polynomials $\mathrm{CX}_{n}(d)$ up to $n=10$.

Let us write $\mathrm{CX}_{n}(d)=\sum_{i=0}^{n-2} a_{n, i} d^{n-i-1}$. (There is no constant term since zero is a root of $\mathrm{CX}_{n}(d)$.) It turns out that there are formulae for the sequences of coefficients of these polynomials. A direct consequence of the proof of Theorem 8 is that the value of the $n$th element of $\left(a_{n, 0}\right)=(1,1,2,16 / 3,50 / 3, \ldots)$, the sequence of leading coefficients in $\mathrm{CX}_{n}(d)$, is

$$
a_{n, 0}=\mathrm{DX}(n, n-1) /(n-1) !=2^{n-1} n^{n-3} /(n-1) ! \text {. }
$$

From Lunnon's formula (1) we can infer the value of the $n$th element of the sequence of second coefficients of the polynomials $\mathrm{CX}_{n}(d),(0,0,-1,15 / 2,-42, \ldots)$, by summing up the 
coefficients of $d^{n-2}$ :

$$
\begin{aligned}
a_{n, 1} & =\operatorname{DX}(n, n-1) \frac{\sum_{i=0}^{n-2}(-i)}{(n-1) !}+\operatorname{DX}(n, n-2) \frac{1}{(n-2) !} \\
& =2^{n-1} n^{n-3} \frac{-1}{2(n-3) !}+2^{n-3} n^{n-5}(n-2)\left(2 n^{2}-6 n+9\right) \frac{1}{(n-2) !} \\
& =-3 \cdot 2^{n-3} n^{n-5}(2 n-3) /(n-3) !
\end{aligned}
$$

Here we use the convention $0 !=1$ and $1 /(-1) !=1 /(-2) !=0$, and so $a_{1,1}=a_{2,1}=0$ and $a_{3,1}=-1$.

Since we also know the formula for $\mathrm{DX}(n, n-3)$, we can, in the same manner, compute

$$
\begin{aligned}
a_{n, 2}= & \operatorname{DX}(n, n-1) \frac{\sum_{0 \leq i<j \leq n-2} i j}{(n-1) !}+\operatorname{DX}(n, n-2) \frac{\sum_{i=0}^{n-3}(-i)}{(n-2) !}+\operatorname{DX}(n, n-3) \frac{1}{(n-3) !} \\
= & \frac{2^{n-1} n^{n-3}(n-1)(n-2)(n-3)(3 n-4)}{24(n-1) !} \\
& \quad-\frac{2^{n-3} n^{n-5}(n-2)\left(2 n^{2}-6 n+9\right)(n-3)(n-2)}{2(n-2) !} \\
& \quad+\frac{2^{n-6} n^{n-7}(n-3)\left(12 n^{5}-104 n^{4}+360 n^{3}-679 n^{2}+1122 n-1560\right)}{3(n-3) !} \\
= & \frac{2^{n-6} n^{n-7}\left(108 n^{3}-463 n^{2}+1122 n-1560\right)}{3(n-4) !},
\end{aligned}
$$

which is the formula of the $n$th element in the sequence $(0,0,0,19 / 6,239 / 6,986 / 3, \ldots)$.

We now compute the limit growth rate of these sequences. We observe, again, the familiar limit

$$
\begin{aligned}
\frac{a_{n+1,0}}{a_{n, 0}} & =\frac{2^{n}(n+1)^{n-2}(n-1) !}{n ! 2^{n-1} n^{n-3}}=2\left(\frac{n+1}{n}\right)^{n-2} \underset{n \rightarrow \infty}{\longrightarrow} 2 e, \\
\frac{a_{n+1,1}}{a_{n, 1}} & =\frac{-3 \cdot 2^{n-2}(n+1)^{n-4}(2 n-1)(n-3) !}{(n-2) !(-3) 2^{n-3} n^{n-5}(2 n-3)} \\
& =\frac{2(2 n-1)(n+1)}{(2 n-3)(n-2)}\left(\frac{n+1}{n}\right)^{n-5} \underset{n \rightarrow \infty}{\longrightarrow} 2 e,
\end{aligned}
$$

and, similarly,

$$
\frac{a_{n+1,2}}{a_{n, 2}} \underset{n \rightarrow \infty}{\longrightarrow} 2 e
$$

Note the resemblance between the formulae of $a_{n, i}$ (for $\left.i=0,1,2\right)$. We conjecture that for all $i \geq 1$ we have $a_{n, i}=(-1)^{i} 2^{n-2 i-1} n^{n-2 i-3} P_{i}(n) /(n-i-2)$ !, where $P_{i}(n)$ is a polynomial in $n$, and, thus, $a_{n+1, i} / a_{n, i} \underset{n \rightarrow \infty}{\longrightarrow} 2 e$.

In summary, we know for sure the formulae for $a_{n, 0}, a_{n, 1}$, and $a_{n, 2}$. Based of the conjectured formulae for $\operatorname{DX}(n, n-i)(4 \leq i \leq 6)$, we could obtain, with considerable effort, conjectured formulae for $a_{n, i}(3 \leq i \leq 5)$. In the next section we use the sequences $a_{n, i}$; fortunately, knowing $a_{n, i}(0 \leq i \leq 2)$ is sufficient for obtaining the main result. 


\section{Columns, or: Growth Rate}

In this section we show that the asymptotic growth rate of $d$-dimensional polycubes is linear in $d$, and provide a good estimate of it. We prove rigorously that as $d$ tends to infinity, $\lambda_{d}$ approaches $2 e d-o(d)$, and show, in a semi-rigorous computation, that the asymptotic value is $(2 d-3) e$. As mentioned in the introduction, this was already argued in the statistical-physics literature, where, based on the unproven assumption that $\operatorname{CX}(n, d) \sim C_{d} n^{\theta} \lambda_{d}^{n}$ (where $\theta$ is widely believed to be equal to -1$)$, and using the unproven diagonal formulae for $\mathrm{DX}(n, n-i)$ $(i=2,3)$, a $1 / d$-expansion was computed for $\ln \lambda_{d}$. We show that this representation of $\lambda_{d}$ supports our estimate.

First, we show an upper bound:

Theorem 9. For any fixed value of $d, \lambda_{d}=\lim _{n \rightarrow \infty} \sqrt[n]{\operatorname{CX}(n, d)}<(2 d-1) e$.

Proof. We generalize an idea of Eden [Ed61], who proved the bound $\lim _{n \rightarrow \infty} \sqrt[n]{\mathrm{CX}(n, 2)} \leq$ 6.75 in the plane. (Klarner and Rivest [KR73, §2] applied a more complex version of this technique in two dimensions, improving the upper bound to 4.65. Whittington and Soteros [WS90, §2] use a similar argument for $d$-dimensional bond animals.) We demonstrate that $\mathrm{CX}(n, d) \leq\left(\begin{array}{c}(2 d-1) n \\ n-1\end{array}\right)$ by constructing an injection from polycubes of size $n$ to $(n-1)$-element subsets of a set whose cardinality is $(2 d-1) n$. Given a polycube $P$, assign the numbers 1 through $n$ to the cubes of $P$ in lexicographic order (defined by the coordinates of the centers of the cubes in $d$-space). Now perform a breadth-first search on the cube-connectivity graph $G$ of $P$, again, in lexicographic order, starting from cube 1 . In the course of this procedure, any cube $c \in P$ (except cube 1) is reached through an incoming edge $e$. (An imaginary edge incoming into cube 1 is fixed so as to supposedly originate from a cube that cannot belong to any polycube. This is possible due to the lexicographic order of the cubes.) The cube $c$ is connected by edges of $G$ to at most $2 d-1$ additional neighboring cubes. We label each of the outgoing edges leading to these neighbors with a pair of numbers $(i, j)$. The value $i$ is the number associated with $c$. The value $1 \leq j \leq 2 d-1$ is determined by the orientation of the outgoing edge relative to $e$. In two dimensions, for example, the outgoing edges are numbered according to their clockwise order relative to $e$. By the end of the search, each of the $n-1$ edges of the resulting spanning tree is given a unique label from a set of $(2 d-1) n$ possible labels. Let us argue why this is an injection. Apply the search procedure to two different polycubes, and let $c$ be the first cube (during the search) in which a discrepancy in neighboring cubes occurs. This will immediately result in a corresponding label discrepancy, which the search will not be able to "remedy" later because it will never visit $c$ again.

Furthermore, it is easily verified that

$$
\lim _{n \rightarrow \infty} \sqrt[n]{\left(\begin{array}{c}
(2 d-1) n \\
n-1
\end{array}\right)}=(2 d-1)\left(1+\frac{1}{2(d-1)}\right)^{2(d-1)}<(2 d-1) e .
$$

Second, we show a lower bound. The asymptotic bound, as $d$ tends to infinity, almost matches the upper bound proven in Theorem 9. 
Theorem 10. $\lim _{d \rightarrow \infty} \lambda_{d} / d \geq 2 e$.

Proof. Our goal is to show that $\lim _{d \rightarrow \infty} \lim _{n \rightarrow \infty} \sqrt[n]{\mathrm{CX}(n, d)} / d \geq 2 e$. Fix $\varepsilon>0$. It can easily be verified from (3) that the sequence $\sqrt[n-1]{a_{n, 0}}$ converges to $2 e$. Therefore, there exists $n_{0}=n_{0}(\varepsilon)$ large enough and independent of $d$, so that $\sqrt[n_{0}-1]{a_{n_{0}, 0}} \geq(2-\varepsilon / 2) e$. According to Theorem $8, \mathrm{CX}_{n_{0}}(d)$ is a polynomial in $d$ of degree $n_{0}-1$. Thus, $\lim _{d \rightarrow \infty}\left(\sqrt[n_{0}-1]{\mathrm{CX}_{n_{0}}(d)} / d\right)=$ $\lim _{d \rightarrow \infty}\left(\sqrt[n_{0}-1]{a_{n_{0}, 0} d^{n_{0}-1}} / d\right)=\sqrt[n_{0}-1]{a_{n_{0}, 0}} \cdot 2$ Hence, there exists $d_{0}=d_{0}\left(n_{0}\right)$ such that for all $d \geq d_{0}$ we have $\left(\sqrt[n_{0}-1]{\mathrm{CX}_{n_{0}}(d)}\right) / d \geq(2-\varepsilon) e$, that is,

$$
\sqrt[n_{0}-1]{\mathrm{CX}\left(n_{0}, d\right)} \geq(2-\varepsilon) e d .
$$

Now consider any fixed value of $d \geq d_{0}$. A standard polycube-concatenation argument (superadditivity of $\log \mathrm{CX}(n, d), d$ fixed) shows that $\lambda_{d}=\lim _{n \rightarrow \infty} \sqrt[n-1]{\mathrm{CX}(n, d)} \geq \sqrt[n^{*}-1]{\mathrm{CX}\left(n^{*}, d\right)}$ for any $n^{*}$, in particular, for $n^{*}=n_{0}$. (Instead of attaching polycubes, we identify the two anchor cubes.) Together with (4), this implies that $\lambda_{d} \geq(2-\varepsilon) e d$ for any $d \geq d_{0}$. This establishes the claim.

Note that the main difficulty in the above proof is to justify the exchange of limits in the term $\lim _{d \rightarrow \infty} \lim _{n \rightarrow \infty} \sqrt[n]{\mathrm{CX}(n, d)} / d$. If we exchange the order of limits, the claim that this quantity is at least $2 e$ becomes straightforward. In a beautiful discussion of this issue, Gaunt and Peard [GP00, p. 7524] mention that "in the formal procedure normally used, the order of limits is interchanged," and explain why this operation causes the $1 / d$-expansion to sometimes fail. In some models, e.g., that of self-avoiding walks, it is known [HS95] that this interchange of limits is allowed. In other models, e.g., that of strongly-embedded site animals, one must justify rigorously why this interchange is permissable. Also note that the above proof could not use the sequence $\mathrm{CX}\left(n_{0}+1, d\right) / \mathrm{CX}\left(n_{0}, d\right)$ since it has never been proven that this sequence is monotone increasing, or, at least, that it fully lies below its limit. Finally, note that the proof depends only on the formula (3) for $a_{n, 0}$, while the exact formulae for $a_{n, i}, i>0$, are insignificant.

Combining Theorems 9 and 10, we obtain our main result.

Theorem 11. $\lambda_{d}=2 e d-o(d)$ as $d \rightarrow \infty$.

As mentioned above, this has already been mentioned in statistical physics, but has never been proven rigorously. We now make a more precise conjecture on the asymptotic value of $\lambda_{d}$. We know exactly the first three coefficients of the polynomial $\mathrm{CX}_{n}(d)=a_{n, 0} d^{n-1}+$ $a_{n, 1} d^{n-2}+a_{n, 2} d^{n-3}+\cdots$. Considering $n$ as a fixed number, we can estimate the terms

\footnotetext{
${ }^{2}$ Note that the argument breaks here if we take the $n_{0}$ th root instead of the $\left(n_{0}-1\right)$ st root.
} 
$\mathrm{CX}_{n+1}(d) / \mathrm{CX}_{n}(d)$ and $\sqrt[n]{\mathrm{CX}_{n}(d)}$ as power series in $1 / d$. First,

$$
\begin{aligned}
\frac{\mathrm{CX}_{n+1}(d)}{\mathrm{CX}_{n}(d)}= & \frac{a_{n+1,0}}{a_{n, 0}} d+\frac{a_{n+1,1} a_{n, 0}-a_{n+1,0} a_{n, 1}}{\left(a_{n, 0}\right)^{2}} \\
& +\frac{a_{n, 0}\left(a_{n+1,2} a_{n, 0}-a_{n+1,0} a_{n, 2}\right)-a_{n, 1}\left(a_{n+1,1} a_{n, 0}-a_{n+1,0} a_{n, 1}\right)}{\left(a_{n, 0}\right)^{3}} \cdot \frac{1}{d}+O\left(\frac{1}{d^{2}}\right) \\
= & f_{1}(n) d+f_{2}(n)+\frac{f_{3}(n)}{d}+O\left(\frac{1}{d^{2}}\right),
\end{aligned}
$$

where

$$
\begin{aligned}
& f_{1}(n)=2\left(\frac{n+1}{n}\right)^{n-2}, \\
& f_{2}(n)=-\frac{3(n-1)(n+1)^{n-4}\left(2 n^{3}+6 n^{2}-5 n-6\right)}{2 n^{n}}, \text { and } \\
& f_{3}(n)=-\frac{(n-2)(n-1)(n+1)^{n-6}\left(31 n^{6}+217 n^{5}-4888 n^{4}+743 n^{3}+13209 n^{2}+12660 n+3708\right)}{48 n^{n+2}} .
\end{aligned}
$$

It is now easy to verify that $\lim _{n \rightarrow \infty} f_{1}(n)=2 e, \lim _{n \rightarrow \infty} f_{2}(n)=-3 e$, and $\lim _{n \rightarrow \infty} f_{3}(n)=$ $-31 e / 48$. Second,

$$
\begin{aligned}
\sqrt[n]{\mathrm{CX}_{n}(d)} & =\left(a_{n, 0}\right)^{1 / n} d+\frac{a_{n, 1}}{n\left(a_{n, 0}\right)^{(n-1) / n}}-\frac{(n-1)\left(a_{n, 1}\right)^{2}-2 n a_{n, 0} a_{n, 2}}{2 n^{2}\left(a_{n, 0}\right)^{(2 n-1) / n}} \cdot \frac{1}{d}+O\left(\frac{1}{d^{2}}\right) \\
& =g_{1}(n) d+g_{2}(n)+\frac{g_{3}(n)}{d}+O\left(\frac{1}{d^{2}}\right)
\end{aligned}
$$

where

$$
\begin{aligned}
& g_{1}(n)=\left(\frac{2^{n-1} n^{n-3}}{(n-1) !}\right)^{1 / n}, \\
& g_{2}(n)=-\frac{3(n-1)(n-2)(2 n-3)}{4 n^{3}} \cdot\left(\frac{2^{n-1} n^{n-3}}{(n-1) !}\right)^{1 / n}, \text { and } \\
& g_{3}(n)=-\frac{(n-1)(n-2)\left(31 n^{4}-432 n^{3}+2118 n^{2}-2817 n-486\right)}{96 n^{6}} \cdot\left(\frac{2^{n-1} n^{n-3}}{(n-1) !}\right)^{1 / n}
\end{aligned}
$$

Again, $\lim _{n \rightarrow \infty} g_{1}(n)=2 e, \lim _{n \rightarrow \infty} g_{2}(n)=-3 e$ and $\lim _{n \rightarrow \infty} g_{3}(n)=-31 e / 48$. Assuming without proof that the limits $n \rightarrow \infty$ and $d \rightarrow \infty$ can be exchanged and yield a converging series leads us to the conjecture that

$$
\lambda_{d}=2 e d-3 e-\frac{31 e}{48 d}+O\left(\frac{1}{d^{2}}\right)
$$

and, thus, $\lambda_{d}$ tends to $(2 d-3) e$ as $d$ tends to infinity.

This is consistent with the unproven $1 / d$-expansion of the free energy (the logarithm of the growth constant) found in various publications of Gaunt et al., the most general of 
which, that we are aware of, is Equation (3.8) in [GP00, p. 7521]. By substituting $y:=1$ and $z:=0$ in it, we obtain the expansion [ibid., p. 7538 , see $F^{(d)}(y ; C)$ ]

$$
\ln \lambda_{d}=\ln \sigma+1-\frac{2}{\sigma}-\frac{79}{24 \sigma^{2}}+O\left(\frac{1}{\sigma^{3}}\right)
$$

where $\sigma=2 d-1$. A common estimate is achieved by truncating this expansion after the second term and writing (see, e.g., [GP00, p. 7523, Eq. 4.9]) $\ln \lambda_{d} \sim \ln (2 d-1)+1$, leading to the inaccurate conclusion $\lambda_{d} \sim(2 d-1) e$. This estimate is also known as $\lambda_{B}$, the "Bethe approximation" of $\lambda$. Refer, for example, to Equation (3.9) in [GSR76, p. 1904], which states that $\lambda_{B}=\sigma^{\sigma} /(\sigma-1)^{\sigma-1}$. Substituting $\sigma=2 d-1$ and taking $d$ to infinity, one can verify that $\lambda_{B}$ tends to $(2 d-1) e$. In fact, one or two more terms from Equation (6) are needed in order to estimate the asymptotic value more accurately. It is easily seen that

$$
\begin{aligned}
\lambda_{d} & \sim(2 d-1) \cdot e \cdot e^{-2 /(2 d-1)} \cdot e^{-79 /\left(24(2 d-1)^{2}\right)} \\
& =(2 d-1) e\left(1-\frac{2}{(2 d-1)}+\frac{2}{(2 d-1)^{2}}+O\left(\frac{1}{d^{3}}\right)\right)\left(1-\frac{79}{24(2 d-1)^{2}}+O\left(\frac{1}{d^{4}}\right)\right) \\
& =(2 d-3) e-\frac{31}{48 d}+O\left(\frac{1}{d^{2}}\right),
\end{aligned}
$$

which is in agreement with our conjecture (5).

Finally, we show that the result about the asymptotic growth rate of all polycubes applies also to proper polycubes. In two dimensions, this follows easily from the fact that $\mathrm{DX}(n, 2)=$ $\mathrm{CX}(n, 2)-2$ (only the two "sticks" are improper). It is still rather easy to prove a similar result in $d$ dimensions.

Theorem 12. $\lim _{n \rightarrow \infty} \operatorname{DX}(n+1, d) / \operatorname{DX}(n, d)=\lim _{n \rightarrow \infty} \sqrt[n]{\operatorname{DX}(n, d)}=\lambda_{d}$

Proof. We will show that there exists a constant $c_{d}$ that depends only on $d$, such that $c_{d} \mathrm{CX}(n, d)<\mathrm{DX}(n, d)$. Due to Madras [Ma99], $\lim _{n \rightarrow \infty} \mathrm{CX}(n, d) / \mathrm{CX}(n-d-1, d)$ exists and equals $\lambda_{d}^{d+1}$. Thus, for almost all $n$, we have $\operatorname{CX}(n, d)<\left(\lambda_{d}^{d+1}+1\right) \operatorname{CX}(n-d-1, d)$. In addition, $\mathrm{CX}(n-d-1, d) \leq \mathrm{DX}(n, d)$ by concatenating an arbitrarily-chosen proper $d$ dimensional polycube of size $d+1$ with all polycubes of size $n-d-1$ in $d$ dimensions. Hence, $c_{d}=1 /\left(\lambda_{d}^{d+1}+1\right)$ is an appropriate constant. Since, in addition, $\operatorname{DX}(n, d)<\operatorname{CX}(n, d)$, and by applying the squeezing theorem, it follows that $\lim _{n \rightarrow \infty} \sqrt[n]{\mathrm{DX}(n, d)}=\lambda_{d}$. A "Madras-like" pattern argument completes the proof.

\section{Conclusion}

We have proved in this paper, for the first time, several formulae for polycubes in higher dimensions. We have also shown that the asymptotic growth rate of polycubes in $d$ dimensions approaches $2 e d-o(d)$ as $d$ tends to infinity. We conjecture that the asymptotic growth rate is $(2 d-3) e$. 


\section{References}

[AB06] G. Aleksandrowicz and G. Barequet, Counting $d$-dimensional polycubes and nonrectangular planar polyominoes, Proc. 12th Ann. Int. Computing and Combinatorics Conf., Taipei, Taiwan, Lecture Notes in Computer Science, 4112, Springer-Verlag, 418-427, August 2006. Full version to appear in Int. J. of Computational Geometry and Applications.

[AB08] G. Aleksandrowicz and G. Barequet, Counting polycubes without the dimensionality curse, Proc. 14th Ann. Int. Computing and Combinatorics Conf., Dalian, China, Lecture Notes in Computer Science, 5092, Springer-Verlag, 100-109, June 2008. Full version to appear in Discrete Mathematics.

[BMrR06] G. Barequet, M. Moffie, A. Ribó, and G. Rote, Counting polyominoes on twisted cylinders, Integers (electronic journal), 6 (2006), \#A22, 37 pp.

[BH57] S.R. Broadbent and J.M. Hammersley, Percolation processes: I. Crystals and mazes, Proc. Cambridge Philosophical Society, 53 (1957), 629-641.

[Ed61] M. Eden, A two-dimensional growth process, Proc. 4th Berkeley Symp. on Mathematical Statistics and Probability, IV, Berkeley, CA, 223-239, 1961.

[Ga80] D.S. Gaunt, The critical dimension for lattice animals, J. of Physics A: Mathematical and General, 13 (1980), L97-L101.

[GP00] D.S. Gaunt And P.J. Peard, $1 / d$-expansions for the free energy of weakly embedded site animal models of branched polymers, J. of Physics A: Mathematical and General, 33 (2000), $7515-7539$.

[GPSW94] D.S. Gaunt, P.J. Peard, C.E. Soteros, and S.G. Whittington, Relationships between growth constants for animals and trees, J. of Physics A: Mathematical and General, 27 (1994), $7343-7351$.

[GSR76] D.S. Gaunt, M.F. Sykes, And H. Ruskin, Percolation processes in $d$-dimensions, J. of Physics A: Mathematical and General, 9 (1976), 1899-1911.

[HS95] T. Hara AND G. Slade, The self-avoiding-walk and percolation critical points in high dimensions, Combinatorics, Probability, and Computing, 4 (1995), 197-215.

[Je03] I. Jensen, Counting polyominoes: A parallel implementation for cluster computing, Proc. Int. Conf. on Computational Science, part III, Melbourne, Australia and St. Petersburg, Russia, Lecture Notes in Computer Science, 2659, Springer, 203-212, June 2003.

[K167] D.A. Klarner, Cell growth problems, Canadian J. of Mathematics, 19 (1967), 851-863.

[KR73] D.A. Klarner And R.L. Rivest, A procedure for improving the upper bound for the number of n-ominoes, Canadian J. of Mathematics, 25 (1973), 585-602.

[Lu72] W.F. Lunnon, Symmetry of cubical and general polyominoes, in: Graph Theory and Computing (R.C. Read, ed.), Academic Press, New York, 1972, 101-108.

[Lu75] W.F. Lunnon, Counting multidimensional polyominoes, The Computer Journal, 18 (1975), $366-367$.

[Ma99] N. Madras, A pattern theorem for lattice clusters, Annals of Combinatorics, 3 (1999), 357384 . 
[MSW+90] N. Madras, C.E. Soteros, S.G. Whittington, J.L. Martin, M.F. Skeys, S. FleSIA, AND D.S. Gaunt, The free energy of a collapsing branched polymer, J. of Physics A: Mathematical and General, 23 (1990), 5327-5350.

[Ma90] J.L. MARTin, The impact of large-scale computing on lattice statistics, J. of Statistical Physics, 58 (1990), 749-774.

[Mo70] J.W. Moon, Counting Labelled Trees, Canadian Mathematical Monographs, no. 1, Wiliam Clowes \& Sons, London and Beccles, 1970.

[PG95] P.J. PEard And D.S. Gaunt, 1/d-expansions for the free energy of lattice animal models of a self-interacting branched polymer, J. Phys. A: Math. Gen., 28 (1995), 6109-6124.

[Re81] D.H. RedelmeIER, Counting polyominoes: Yet another attack, Discrete Mathematics, 36 (1981), 191-203.

[Ri90] T.J. Rivlin, Chebyshev Polynomials: From Approximation Theory to Algebra and Number Theory (2nd ed.), Wiley, New York, 1990.

[SE05] M. Schwartz And T. Etzion, Two-dimensional cluster-correcting codes, IEEE Trans. on Information Theory, 51 (2005), 2121-2132.

[Sl] N.J.A. SloAne, The on-line encyclopedia of integer sequences, available at http://www. research. att.com/ njas/sequences.

[WS90] S. Whittington And C. Soteros, Lattice animals: Rigorous results and wild guesses, in: Disorder in Physical Systems (G. Grimmett and D. Welsh, eds.), Clarendon Press, Oxford, 1990, 323-335. 